\title{
Neural Systems Underlying the Recognition of Familiar and Newly Learned Faces
}

\author{
Catherine L. Leveroni, ${ }^{1,2}$ Michael Seidenberg, ${ }^{1}$ Andrew R. Mayer, ${ }^{1,2}$ Larissa A. Mead, ${ }^{2}$ Jeffrey R. Binder, ${ }^{2}$ and \\ Stephen M. Rao' \\ 1Department of Psychology, Finch University of Health Sciences/The Chicago Medical School, North Chicago, Illinois \\ 60064, and 2Department of Neurology, Medical College of Wisconsin, Milwaukee, Wisconsin 53226
}

\begin{abstract}
Memory for famous faces can be used to examine the neural systems underlying retrieval from long-term memory. To date, there have been a limited number of functional neuroimaging investigations examining famous face recognition. In this study, we compared recognition of famous faces to recognition of newly learned faces. Whole-brain, event-related functional magnetic resonance imaging was used to image regional changes in neural activity in 11 subjects during the encoding of unfamiliar faces and during familiarity judgments for: (1) newly learned faces, (2) unfamiliar face distractors, and (3) famous faces. Image analyses were restricted to correct recognition trials. Recognition accuracy and response time to famous and recently learned faces were equivalent. Recognition of famous
\end{abstract}

faces was associated with a widespread network of bilateral brain activations involving the prefrontal, lateral temporal, and mesial temporal (hippocampal and parahippocampal regions) regions compared to recognition of recently encoded faces or unfamiliar faces seen for the first time. Findings are discussed in relation to current proposals concerning the neural regions thought to participate in long-term memory retrieval and, more specifically, in relation to retrieval of information from the person identity semantic system.

Key words: event-related fMRl; famous faces; frontal; temporal; hippocampus; recognition memory; person-identity system; memory; face recognition; temporofrontal cortex
The successful retrieval of information from long-term memory requires the integrated activity of multiple brain regions. Current proposals, based primarily on findings from the human lesion literature, have focused on the role of the hippocampus and the temporofrontal region in long-term memory retrieval (Squire and Alvarez, 1995; Nadel and Moscovitch, 1997; Kroll et al., 1997). The specific neural system engaged varies with the nature of the stimuli examined (verbal or nonverbal), the time frame of initial acquisition (recent or remote), and the type of information retrieved from long-term memory (personal or public). In the human lesion literature, the recognition and identification of famous faces has commonly been used to study the neural regions critical for retrieval of information from long-term memory (Warrington and James, 1967; Marslen-Wilson and Teuber, 1975; Albert et al., 1979; Warrington and McCarthy, 1988; Barr et al., 1990; Greene and Hodges, 1996; Rempel-Clower et al., 1996).

It is generally acknowledged that famous faces produce automatic retrieval of person-identity information from long-term memory (Bruce and Young, 1986; Burton et al., 1990). Thus, the comparison of famous and unfamiliar faces provides an opportunity to examine the neural systems activated when pre-existing semantic and biographical information is available for retrieval.

\footnotetext{
Received June 15, 1999; revised Oct. 18, 1999; accepted Oct. 18, 1999.

This work was supported by grants from the National Institute of Mental Health (P01-MH51358 and R01-MH57836), National Institute of Neurological Disorders and Stroke (R01-NS33576 and RO1-NS37738), and National Institute of Drug Abuse (R01-DA09465). We thank P. Bellgowan, R. Cabeza, R. Cox, J. Cunningham, S. Fuller, T. Hammeke, J. Hyde, K. Paller, M. Parsons, T. Prieto, A. Rosen, K. Rowe, E. Stein, B. Ward, and S. Woodley for technical assistance and helpful comments.

Correspondence should be addressed to Dr. Stephen M. Rao, Section of Neuropsychology, Medical College of Wisconsin, 9200 West Wisconsin Avenue, Milwaukee, WI 53226. E-mail: srao@mcw.edu.

Copyright (C) 2000 Society for Neuroscience $0270-6474 / 00 / 200878-09 \$ 15.00 / 0$
}

Neuroimaging studies of memory for unfamiliar faces have demonstrated right hippocampal activation during encoding, but no hippocampal activity during subsequent recognition (Grady et al., 1995; Haxby et al., 1996; Clark et al., 1998). Memory for unfamiliar faces has also produced frontal activation, although the side of activation has differed across studies. Haxby et al. (1996) found left frontal activation during encoding, whereas Kelley et al. (1998) observed increased right frontal activation.

Three PET studies of famous face recognition have suggested that the retrieval of person-specific semantic information involves more extensive frontotemporal activation than unfamiliar face recognition; however, the results across studies have not been entirely consistent. Sergent et al. (1992) found that occupation categorization (actor/not actor) produced activation of the right parahippocampal area and bilateral activation in the anterior temporal and orbitofrontal regions. In contrast, left hippocampal activity was observed in another categorization task involving famous faces (Kapur et al., 1995). More recently, Tempini et al. (1998) found extensive left-sided activation of the frontal and temporal regions with no increased activity in the medial temporal region when subjects were asked to match famous faces. The divergent results may be attributable in part to differences in face processing (matching vs categorization) and control (rest vs gender classification) tasks.

We present findings from a whole-brain, event-related functional magnetic resonance imaging (fMRI) study that directly compared the recognition of newly learned, unfamiliar faces with the recognition of well known faces. This study was designed to contrast activation patterns resulting from: (1) famous face recognition with recognition of newly learned faces and unfamiliar faces not previously encountered, and (2) newly learned unfamil- 
iar faces with those for unfamiliar faces not been previously encoded.

\section{MATERIALS AND METHODS}

Subjects. Eleven healthy, right-handed volunteers (five male and six female; mean age, 32.0; range, 25-36) were studied. Subjects were all strongly right-handed (mean laterality quotient, 97.5; range, 88-100) on the Edinburgh Handedness Inventory (Oldfield, 1971). Subjects were excluded if they had a history of neurological disease, major psychiatric disturbance, substance abuse, or were taking psychoactive prescriptive medications. Informed consent was obtained from subjects according to institutional guidelines established by the Medical College of Wisconsin Human Subjects Review Committee. Subjects were compensated for their time.

Experimental task. The stimuli consisted of 150 black and white photos of adult faces (e.g., models) digitally scanned from books and magazines. Luminance and contrast were adjusted to make the images as comparable as possible; dimensions of the photos were interpolated to $240 \times 240$ pixels. One hundred of the faces were unfamiliar. Male and female faces were equally represented and covered a wide age distribution. Half of the unfamiliar faces $(n=50)$ were seen twice; once during both the encoding and retrieval conditions (see below). The other half $(n=50)$ were used as foils and were seen only one time during the retrieval condition. The remaining 50 faces consisted of pictures of well-known entertainers, politicians, and sports figures. As in the unfamiliar faces, males and females were equally represented and covered a wide age range. The famous faces were drawn from a time period that spanned the 1970s, 1980 s, and early 1990 s. During the inactive period between face presentations, subjects were asked to maintain visual fixation on a small centrally located black box presented within a scrambled face. The scrambled image provided control for luminance of the visual display. A trial consisted of a single face stimulus presentation for $2.5 \mathrm{sec}$ followed by a $12.5 \mathrm{sec}$ visual fixation period.

All visual stimuli were computer-generated and rear-projected onto the center of an opaque screen located at the subject's feet (viewing distance, $200 \mathrm{~cm}$ ). Face and fixation stimuli subtended a $4 \times 4^{\circ}$ visual angle. Subjects viewed the screen in a darkened room through prism glasses and corrective lenses, if necessary. A nonferrous keypress device made from force-sensing resistors was used to record response times and accuracy.

For the encoding (EN) condition, 50 unfamiliar faces were presented in random order. Subjects were instructed to remember the faces because they would later be asked to identify them. To aid with encoding, subjects were asked to make a judgment about the pleasantness of the face to enhance recognition memory performance. Subjects recorded their judgment by pressing one of two keys with the right index finger. Subjects pressed the left key if the face was judged to be "pleasant" and the right key if the face was "unpleasant." Stimuli were presented in two imaging runs of 25 trials each.

The retrieval condition began 12 min after the completion of the second encoding imaging run. For this condition, 150 faces from the three stimulus conditions [i.e., famous faces (FF), newly learned (NL) faces, and foils (FO)] were randomly divided into six functional imaging runs ( 25 trials per run). Within each run, faces from the three stimulus conditions were ordered in a pseudorandom fashion. Subjects were instructed that one-third of the faces would be famous, another third would be faces they had seen previously during the encoding phase, and the final third were new faces. Subjects pressed one of two keys with their right index finger. If they had seen the face before, i.e., newly learned or famous, the left button was to be pressed; if the face was judged to be new, i.e., never seen before, subjects pressed the right key. Accuracy and response latency to the first keypress were recorded.

Duration of each of the eight imaging runs (two encoding, six retrieval) was $6 \mathrm{~min}, 30 \mathrm{sec}$.

Functional MRI. Whole-brain, event-related functional MRI was conducted on a commercial 1.5 Tesla scanner (Signa; General Electric Medical Systems, Milwaukee, WI) equipped with a three-axis local gradient head coil and an elliptical endcapped quadrature radiofrequency coil (Medical Advances, Milwaukee, WI). Echoplanar images were collected using a single-shot, blipped, gradient-echo echoplanar pulse sequence [echo time (TE), $40 \mathrm{msec}$; field of view (FOV), $24 \mathrm{~cm}$; matrix size, $64 \times 64]$. For the encoding and retrieval conditions, 17 contiguous sagittal 7-mm-thick slices were selected to provide coverage of the entire brain (voxel size, $3.75 \times 3.75 \times 7 \mathrm{~mm}$ ). The interscan interval [repetition time (TR)] was $2.5 \mathrm{sec}$. During each imaging series,
156 sequential echoplanar images were collected. During the period between the encoding and retrieval run, high-resolution, threedimensional spoiled gradient-recalled at steady-state (SPGR) anatomic images were collected [TE, $5 \mathrm{msec}$; TR, $24 \mathrm{msec}$; $40^{\circ}$ flip angle; number of excitations (NEX), 1; slice thickness, 1.2 or $1.3 \mathrm{~mm}$; FOV, $24 \mathrm{~cm}$; resolution, $256 \times 192$ ]. Foam padding was used to limit head motion within the coil.

Image processing and statistical analysis. Each image time series was spatially registered in-plane to reduce the effects of head motion using an iterative linear least-squares method (Keren et al., 1988). Functional images were created by subtracting a local baseline (fixation) image from an activation image on a trial-by-trial basis. Specifically, each $15 \mathrm{sec}$ trial consisted of six images. The first and sixth image ( 0 and $12.5 \mathrm{sec}$ after stimulus onset, respectively) in each trial were averaged and referred to as the local baseline image. The third and fourth images (5.0 and $7.5 \mathrm{sec}$ after stimulus onset) were averaged to represent the peak evoked change in the hemodynamic response and referred to as the activation image. The second and fifth images (2.5 and $10.0 \mathrm{sec}$ after stimulus onset) were not analyzed because they represent the transitional rise and fall of the evoked hemodynamic response (Bandettini et al., 1992). In the next stage, a difference image was created for each trial by subtracting the baseline image from activation image. Thus, a total of 200 difference images were created for each of the four conditions (50 EN, $50 \mathrm{FF}, 50$ $\mathrm{NL}$, and $50 \mathrm{FO}$ ). An average difference image (ADI) was created for each of the four conditions by averaging all of the individual difference images within a condition. For the encode condition, the ADI was based on all trials in which the unfamiliar face was correctly identified during subsequent recognition testing (NL condition). For the three retrieval conditions (FF, NL, and FO), only correct trials (recognition of a FF or NL face or rejection of a FO face) were averaged. Thus, for each subject, four ADIs were generated per slice.

Individual SPGR anatomical scans and average difference images were linearly interpolated to volumes with $1 \mathrm{~mm}^{3}$ voxels, co-registered, and transformed into standard stereotaxic space (Talairach and Tournoux, 1988) using the Medical College of Wisconsin Analysis of Functional Neuroimages software package (Cox, 1996). Functional images were blurred using a $4 \mathrm{~mm}$ Gaussian full-width half-maximum filter to compensate for intersubject variability in anatomic and functional anatomy.

Statistical comparisons were made between the three recognition conditions (FF, NL, and FO), and between the NL and EN conditions. A one-way repeated measures ANOVA was applied to the three retrieval conditions on a voxel-by-voxel basis across the 11 subjects. This was followed by a pooled variance $t$ test to compare each of the conditions in a pairwise fashion (FF vs NL; FF vs FO; and NL vs FO). A cutoff $t$ value of $3.15(p<0.005 ; \mathrm{df}=10)$ was established as the threshold for significance. The NL and EN conditions were compared with a paired $t$ test using the same probability threshold $(t=3.58 ; p<0.005 ; \mathrm{df}=10)$ as the retrieval comparisons. A minimum cluster size threshold of $0.2 \mathrm{ml}$ was applied to minimize false-positive activation foci from the brain maps.

\section{RESULTS}

\section{Behavioral data}

Figure $1 A$ presents the overall accuracy for recognition of famous (FF) and newly learned (NL) faces and rejection of foils (FO). Subjects correctly identified $68 \%$ of the famous faces and $75 \%$ of the newly learned faces; percentage correct rejection of foils was 94.7\% with errors attributable to missed responses rather than false positives. All conditions were performed well above chance $(>60 \%)$, except one subject who performed at $50 \%$ on the NL condition and another subject who performed at $46 \%$ on the FF condition. Repeated measures ANOVA revealed a significant difference in accuracy across the three conditions $\left(F_{(2,20)}=22.68\right.$; $p<0.001$ ). Post hoc analyses (Tukey's honest significant difference) indicated no significant difference $(p>0.20)$ in accuracy between famous and newly learned faces. Subjects were significantly more accurate at rejecting a foil than recognizing famous or newly learned faces $(p<0.001)$.

An accuracy/speed trade-off was observed $\left(F_{(2,20)}=5.5 ; p<\right.$ 0.02 ; Fig. $1 B$ ), with reaction time (RT) for rejecting a foil being significantly slower than RT for recognizing either famous or 
A

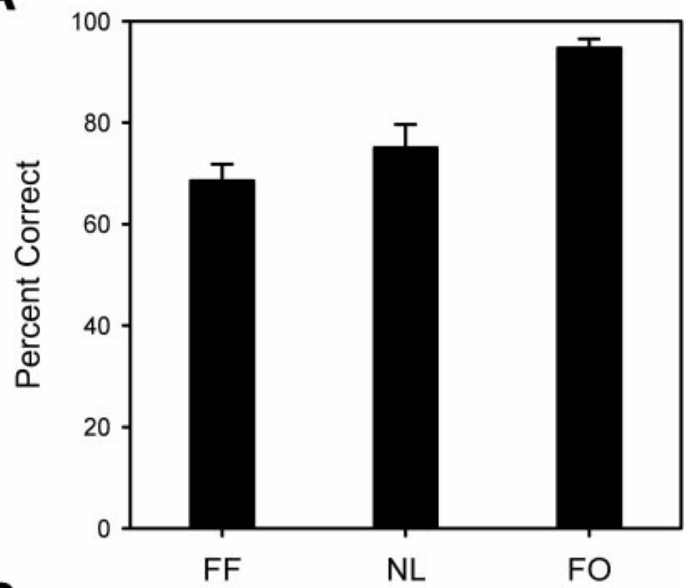

B

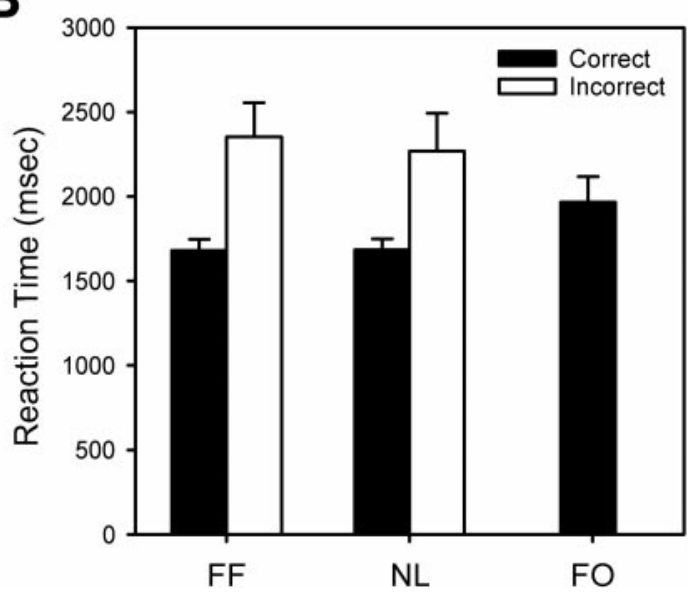

Figure 1. A, Percentage of correct recognition of famous faces $(F F)$ and newly learned $(N L)$ faces and correct rejection of foils $(F O)$. B, Mean of median reaction times to FF, NL, and FO face stimuli. Error bars indicate SEM.

newly learned faces $(p=0.05)$. No differences in RT were observed between correct recognition of famous or newly learned faces $(p>0.20)$.

\section{Activation data}

\section{Famous faces versus newly learned faces}

The comparison of FF and NL faces yielded the greatest number of activated brain regions (Table 1). Recognition of FF stimuli resulted in relatively greater activation than recognition of $\mathrm{NL}$ stimuli. Famous faces produced bilateral activation of the temporal lobes, with the largest areas of activation observed in the anterior middle temporal gyrus. Additional areas of activation extended posteriorly to the temporoparietal junction bilaterally (Fig. 2). Activation of the left hippocampus and right parahippocampal gyrus was also observed (Fig. 3).

Widespread areas of activation for FF were also observed in the prefrontal cortex, including the right anterior cingulate (BA 32), bilateral medial frontal (BA 9, 10,11), bilateral superior frontal (BA 8), and right inferior frontal gyri (BA 47) (Table 1, Fig. 3). Greater activation in the FF condition was also observed in the right and left posterior cingulate/precuneus (BA 23, 30, 31), right anterior inferior parietal (BA 40), and left extrastriate (BA 18) regions. Relative increases in activation $(\mathrm{FF}>\mathrm{NL})$ were also observed bilaterally in the pons and in the right putamen.
Three foci, the right and left posterior inferior parietal (BA 40) and right posterior parietal (BA 7) regions, were significantly more active during recognition of $\mathrm{NL}$ faces than during $\mathrm{FF}$ recognition.

\section{Famous faces versus foils}

FF recognition resulted in greater activation within the temporal lobes relative to the FO stimuli (Table 2, Figs. 2, 3). The areas of activation were very similar to those observed in the FF $>\mathrm{NL}$ comparison (Fig. 2) and included the bilateral middle temporal (BA 21, 39) and right superior temporal (BA 22, 38, 41) areas. In contrast to the comparison with NL faces, activation within the hippocampus was restricted to the right side (Fig. 3). It should be noted however, that increased activity was observed in the left hippocampus in the FF $>$ FO comparison, but just missed our statistical cutoff. No activity was observed within the fusiform area.

Nontemporal lobe activations included a large focus in the left superior frontal cortex (BA 8) along with smaller foci in the right superior frontal (BA 8) and left medial frontal (BA 10) regions. In addition, a large focus was observed in the left posterior cingulate/precuneus area that extended into the homologous region of the right hemisphere.

No areas of significantly increased activation were observed in the foil condition when compared to the famous faces condition.

\section{Newly learned faces versus foils}

Retrieval of newly learned faces resulted in activation of the left medial frontal (BA 6), left inferior parietal (BA 40), and left precuneus (BA 7) regions relative to the foils condition (Table 3 , Fig. 2). It is noteworthy that there were no areas of increased activation within the temporal lobes for this comparison $(\mathrm{NL}>\mathrm{FO})$.

In contrast, rejection of foil stimuli $(\mathrm{FO}>\mathrm{NL})$ resulted in widespread activation of frontal areas bilaterally, including the right medial (BA 6,9), right superior (BA 10), right and left inferior (BA 44, 47), and left precentral (BA 4) areas. As in the FF $>$ NL comparison, the right fusiform area (BA 20/37) was activated.

\section{Encoding versus retrieval of unfamiliar faces}

Encoding of unfamiliar faces resulted in greater activation of the right and left posterior inferior parietal areas (BA 40) relative to recognition of the identical faces (Table 4, Fig. 2). In contrast, NL unfamiliar faces resulted in greater, primarily right-sided, activation within frontal (BA 8, 9, and 6/44), temporal (BA 37), and parietal (BA 40) areas relative to face encoding.

\section{DISCUSSION}

Three primary conclusions can be drawn from this event-related fMRI study. First, recognition of famous faces produced significantly larger MR signal intensity changes over widespread areas of the prefrontal and lateral temporal regions. These changes were observed when famous faces were contrasted with recognition of recently encoded faces or unfamiliar faces seen for the first time. Second, we observed increased activity in hippocampal and parahippocampal regions for famous faces. Third, a recent exposure to a previously unfamiliar face did not produce increased activation of the anterior temporal and hippocampal regions when compared to a novel unfamiliar face. Instead, newly learned faces produced increased activity in frontal and/or parietal regions. These findings are considered in relation to the role and 


\begin{tabular}{|c|c|c|c|c|c|c|}
\hline Loc. \# & Brain region & BA & vol. $(\mathrm{ml})$ & $x$ & $y$ & $z$ \\
\hline & $\mathrm{FF}>\mathrm{NL}$ & & & & & \\
\hline & Frontal Lobe & & & & & \\
\hline 1 & L Superior Frontal & 8 & 2.6 & -15 & 33 & 44 \\
\hline 2 & R Medial Frontal & 9 & 2.4 & 10 & 47 & 25 \\
\hline 3 & R Superior Frontal & 8 & 0.5 & 12 & 40 & 45 \\
\hline 4 & L Medial Frontal & 10 & 0.4 & -6 & 49 & -4 \\
\hline 5 & R Precentral & 6 & 0.4 & 49 & -1 & 13 \\
\hline 6 & L Superior Frontal & 8 & 0.4 & -36 & 15 & 50 \\
\hline 7 & R Inferior Frontal & 47 & 0.3 & 32 & 32 & -7 \\
\hline 8 & $\mathrm{R}$ Anterior Cingulate & 32 & 0.3 & 11 & 21 & -7 \\
\hline 9 & R Medial Frontal & 11 & 0.3 & 9 & 35 & -13 \\
\hline \multirow[t]{2}{*}{10} & L Medial Frontal & 11 & 0.3 & -6 & 39 & -14 \\
\hline & Temporal Lobe & & & & & \\
\hline 11 & L Middle Temporal & 21 & 2.7 & -51 & -11 & -13 \\
\hline 12 & R Middle Temporal & 21 & 1.9 & 52 & -6 & -18 \\
\hline 13 & L Middle Temporal & 21 & 0.6 & -49 & -42 & 7 \\
\hline 14 & L Middle Temporal & 39 & 0.5 & -46 & -68 & 22 \\
\hline 15 & R Superior Temporal & 22 & 0.5 & 54 & -52 & 15 \\
\hline 16 & R Fusiform & $20 / 37$ & 0.4 & 32 & -46 & -16 \\
\hline 17 & R Middle Temporal & 37 & 0.3 & 43 & -64 & 9 \\
\hline 18 & R Insula & - & 0.3 & 37 & 3 & 11 \\
\hline 19 & R Parahippocampal & 35 & 0.2 & 30 & -14 & -23 \\
\hline 20 & R Parahippocampal & 36 & 0.2 & 24 & -43 & -7 \\
\hline \multirow[t]{2}{*}{21} & L Hippocampus & 28 & 0.2 & -19 & -12 & -20 \\
\hline & Parietal/Occipital Lobe & & & & & \\
\hline 22 & L Posterior Cingulate & $23 / 30$ & 1.7 & -4 & -57 & 15 \\
\hline 23 & R Inferior Parietal & 40 & 0.5 & 44 & -30 & 22 \\
\hline 24 & R Posterior Cingulate & 31 & 0.3 & 2 & -57 & 29 \\
\hline \multirow[t]{2}{*}{25} & L Extrastriate & 18 & 0.3 & -20 & -89 & 20 \\
\hline & Subcortical & & & & & \\
\hline 26 & R Pons & - & 0.4 & 11 & -43 & -34 \\
\hline 27 & L Pons & - & 0.2 & -10 & -43 & -33 \\
\hline \multirow[t]{3}{*}{28} & R Putamen & - & 0.3 & 22 & -7 & -6 \\
\hline & $\mathrm{NL}>\mathrm{FF}$ & & & & & \\
\hline & Parietal Lobe & & & & & \\
\hline 29 & L Inferior Parietal & 40 & 1.0 & -37 & -64 & 40 \\
\hline 30 & R Superior Parietal & 7 & 0.5 & 23 & -66 & 30 \\
\hline 31 & R Inferior Parietal & 40 & 0.3 & 35 & -67 & 42 \\
\hline
\end{tabular}

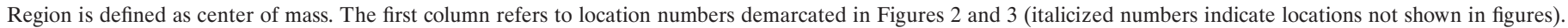
Coordinates represent distance in millimeters from anterior commissure: $x$ right $(+) /$ left $(-) ; y$ anterior $(+) /$ posterior $(-) ; z$ superior $(+) /$ inferior $(-)$.

contribution of the temporofrontal region and hippocampus in long-term memory retrieval.

\section{Temporofrontal activation}

Current models of face processing distinguish between recognition and identification of a face. Face familiarity or recognition can be achieved without also being able to identify a particular face. Person identification requires activation of pre-existing semantic and biographical information stored in long-term memories (Bruce and Young, 1986). Relevant to the current findings, famous faces but not unfamiliar faces (NL or FO) can proceed beyond the stage of recognition to semantic identification (i.e., identity specific code). Recognition of famous faces produced bilateral activation of temporofrontal regions, including the superior, medial, and inferior prefrontal areas, and the anterior to posterior lateral surface of the middle temporal gyrus. In contrast, NL or FO faces did not activate the temporofrontal net- work. It would appear, therefore, that temporofrontal activations are specific to long-term retrieval from the person-identity system rather than face processing and face recognition in general (Nyberg et al., 1996).

Temporofrontal areas are commonly active during semantic retrieval tasks involving words and pictures, although activations are typically left-sided (Vandenberghe et al., 1996; ThompsonSchill et al., 1997; Mummery et al., 1999). Judgment of familiarity for famous faces produced bilateral activation of the temporofrontal system when compared to a similar judgment of familiarity for a recently encountered but "unknown" face. Clinical lesion and neuroimaging studies suggest that the left and right temporal lobes play an important role in retrieving information from the person-identity system (Hanley et al., 1990; Evans et al., 1995; Harris and Kay, 1995; Reinkemeier et al., 1997). Damage to the right anterior temporal area results in selective impairment in 


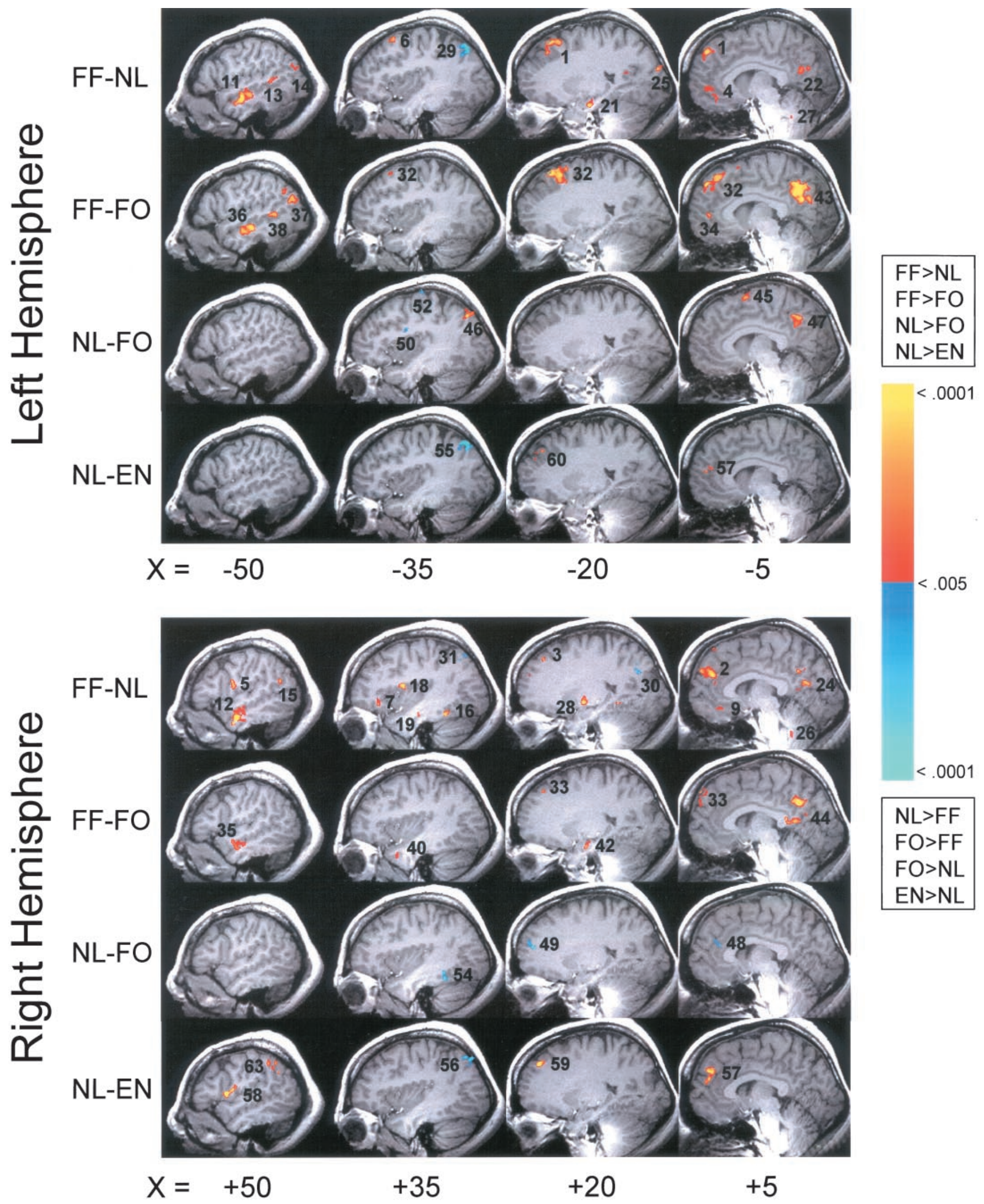

Figure 2. Areas of significantly increased (red-yellow scale) and decreased (blue-cyan scale) MR signal intensity from $t$ tests $(p<0.005)$ comparing the three conditions: FF minus NL, FF minus FO, and NL minus FO. Numbers below each image represent millimeters from the interhemispheric fissure (-, left; + , right). Numbers adjacent to activated foci correspond to location numbers (first column) of Tables 1,2 , and 3. 


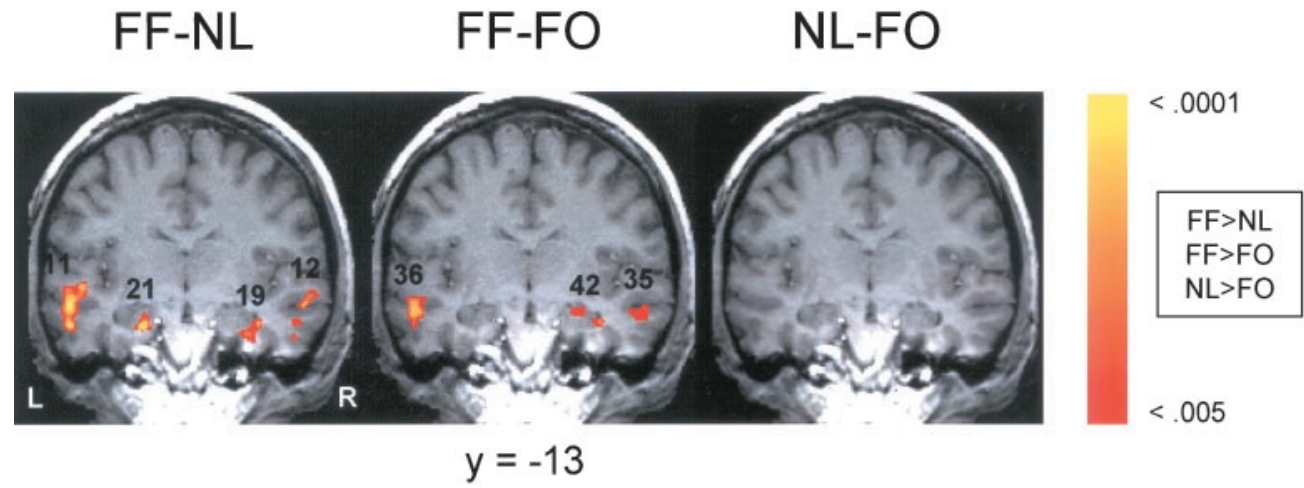

Figure 3. Areas of significant activation within lateral and medial temporal lobes. Number below images indicates millimeters posterior to anterior commissure. Numbers adjacent to activated foci correspond to location numbers (first column) of Tables 1 and 2. semantic identification of famous faces, whereas left anterior temporal damage is associated with impaired identification of animals and tools (Tranel et al., 1997). Similarly, naming people produces bilateral temporal lobe activity on PET imaging, whereas naming tools or animals results in unilateral left temporal lobe activity (Damasio et al., 1996).

Damasio and colleagues (1996) have suggested that the right inferior temporal/polar regions are involved in retrieval of conceptual knowledge about famous faces because they represent "unique" stimuli. Faces provide a means to identify a particular individual on the basis of a unique set of biographical/semantic information (e.g., name, occupation, interests, and place of origin). Well-known faces also have the potential to elicit retrieval of affective responses and personal episodic incidents. Markowitsch and colleagues (Markowitsch, 1995; Kroll et al., 1997) proposed that anterolateral temporal and inferolateral frontal cortices, connected via the uncinate fasciculus, are critical structures supporting retrieval from episodic autobiographical long-term memory. The prefrontal cortex is not the storage site for memory representations, but contributes to memory retrieval by triggering and structuring the search for stored representations, presumably in distributed posterior neocortical sites. Famous faces may intersect both episodic/autobiographical and semantic memory sys- tems and induce more extensive and bilateral temporofrontal activations than other types of stimuli.

\section{Hippocampal activation}

Recognition of famous faces produced increased MR activity in the hippocampal region compared to NL and FO faces. Two PET studies (Sergent et al., 1992; Kapur et al., 1995) found hippocampal activity associated with famous face recognition, but the observed side of activation was inconsistent across the studies. Hippocampal activity also has been reported for long-term retrieval of stimuli other than famous faces. Bilateral hippocampal activation (stronger on the right) was observed for retrieval of navigation information and recalling previously learned routes (Ghaem et al., 1997; Maguire et al., 1997). Maguire and Mummery (1999) examined PET activation associated with recollection of four types of information: autobiographical events, public events, autobiographical facts, and general knowledge. A common retrieval network (predominantly left-sided) was identified that included the anterolateral temporal cortex, parahippocampus, and posterior cingulate. A similar right-sided network, including dorsal prefrontal cortex, was observed when subjects listened to and "imagined" events from autobiographical memory (Fink et al., 1996).

\begin{tabular}{|c|c|c|c|c|c|c|}
\hline Loc. \# & Brain region & BA & vol. (ml) & $x$ & $y$ & $z$ \\
\hline & $\mathrm{FF}>\mathrm{FO}$ & & & & & \\
\hline & Frontal Lobe & & & & & \\
\hline 32 & L Superior Frontal & 8 & 4.5 & -16 & 28 & 46 \\
\hline 33 & R Superior Frontal & 8 & 0.8 & 10 & 49 & 38 \\
\hline \multirow[t]{2}{*}{34} & L Medial Frontal & 10 & 0.4 & -2 & 49 & 4 \\
\hline & Temporal Lobe & & & & & \\
\hline 35 & R Middle Temporal & 21 & 1.4 & 52 & -5 & -16 \\
\hline 36 & L Middle Temporal & 21 & 1.3 & -52 & -15 & -12 \\
\hline 37 & L Middle Temporal & 39 & 1.2 & -50 & -63 & 22 \\
\hline 38 & L Middle Temporal & 21 & 0.6 & -52 & -43 & 4 \\
\hline 39 & R Superior Temporal & $41 / 22$ & 0.3 & 44 & -28 & 17 \\
\hline 40 & R Superior Temporal & 38 & 0.2 & 38 & 10 & -28 \\
\hline 41 & R Superior Temporal & 22 & 0.2 & 58 & -50 & 17 \\
\hline \multirow[t]{2}{*}{42} & R Hippocampus & 28 & 0.4 & 24 & -12 & -17 \\
\hline & Parietal Lobe & & & & & \\
\hline 43 & L Posterior Cingulate & 31 & 5.7 & -2 & -53 & 29 \\
\hline \multirow[t]{3}{*}{44} & R Posterior Cingulate & 29 & 0.8 & 8 & -45 & 7 \\
\hline & $\mathrm{FO}>\mathrm{FF}$ & & & & & \\
\hline & None & & & & & \\
\hline
\end{tabular}




\begin{tabular}{|c|c|c|c|c|c|c|}
\hline Loc. \# & Brain region & BA & vol. (ml) & $x$ & $y$ & $z$ \\
\hline & $\mathrm{NL}>\mathrm{FO}$ & & & & & \\
\hline & Frontal Lobe & & & & & \\
\hline \multirow[t]{2}{*}{45} & L Medial Frontal & 6 & 0.4 & -6 & 6 & 58 \\
\hline & Parietal Lobe & & & & & \\
\hline 46 & L Inferior Parietal & 40 & 0.6 & -37 & -68 & 40 \\
\hline \multirow[t]{3}{*}{47} & L Precuneus & 7 & 0.6 & -5 & -51 & 44 \\
\hline & $\mathrm{FO}>\mathrm{NL}$ & & & & & \\
\hline & Frontal Lobe & & & & & \\
\hline 48 & R Medial Frontal & 9 & 0.5 & 11 & 41 & 25 \\
\hline 49 & R Superior Frontal & 10 & 0.4 & 17 & 52 & 18 \\
\hline 50 & L Inferior Frontal & 44 & 0.3 & -31 & 3 & 23 \\
\hline 51 & R Inferior Frontal & 47 & 0.2 & 30 & 36 & -7 \\
\hline 52 & L Precentral & 4 & 0.2 & -31 & -19 & 61 \\
\hline \multirow[t]{2}{*}{53} & R Medial Frontal & 6 & 0.2 & 15 & -5 & 56 \\
\hline & Temporal Lobe & & & & & \\
\hline 54 & R Fusiform & $20 / 37$ & 0.6 & 32 & -43 & -15 \\
\hline
\end{tabular}

The presence of hippocampal activation for retrieval of information from long-term semantic memory would appear to be inconsistent with the traditional notion that the hippocampus plays a time-limited role in long-term memory retrieval processes (Squire and Alvarez, 1995). However, Cohen et al. (1999) recently proposed that the hippocampus mediates "memory binding or relational memory." From this perspective, famous faces have the potential to be relationally bound to information in preexisting long-term memory (i.e., identification). Similarly, Nadel and Moscovitch (1997) proposed that the mesial temporal lobe acts to update and enrich the semantic network throughout the "life of a memory."

\section{Alternative explanations}

In the current study, activation differences observed for the recognition of FF and NL faces could also be related to dimensions other than familiarity and identification. First, temporofrontal activations during FF retrieval may reflect aspects of face recognition that are unrelated to person-specific semantic memory. Models of face processing propose that the recognition of familiar faces and the matching of unfamiliar faces are subserved by distinct functional pathways that arise from two types of structural encoding. Unfamiliar face processing relies on viewdependent representations, whereas familiar face recognition draws from view-independent representations based on multiple views of the same face (Rolls, 1992; Young et al., 1993). It is conceivable that selective temporofrontal activation during FF recognition may reflect utilization of a unique pathway for the recognition of view-independent representations, rather than retrieval from person-specific semantic networks.

Second, famous face stimuli were presented for the first time during the recognition phase, whereas NL face pictures were previously seen during the encoding phase. Thus, FF and NL faces also differ on the dimension of "novelty" (Martin, 1999). It is important to note, however, that both the famous faces and the foils were seen for the first time (i.e., novel) during the recognition phase. Thus, novelty could be mediating the areas commonly activated by both famous and unfamiliar face foils when compared to previously seen faces. These activations were located

\begin{tabular}{|c|c|c|c|c|c|c|}
\hline Loc. \# & Brain region & BA & vol. $(\mathrm{ml})$ & $x$ & $y$ & $z$ \\
\hline & \multicolumn{6}{|l|}{$\mathrm{EN}>\mathrm{NL}$} \\
\hline & \multicolumn{6}{|l|}{ Parietal Lobe } \\
\hline 55 & L Inferior Parietal & 40 & 0.6 & -36 & -65 & 41 \\
\hline \multirow[t]{3}{*}{56} & R Inferior Parietal & 40 & 0.5 & 36 & -69 & 40 \\
\hline & \multicolumn{6}{|l|}{$\mathrm{NL}>\mathrm{EE}$} \\
\hline & \multicolumn{6}{|l|}{ Frontal Lobe } \\
\hline 57 & R Medial Frontal & 9 & 1.3 & 6 & 45 & 23 \\
\hline 58 & R Precentral & $6 / 44$ & 0.6 & 50 & 5 & 7 \\
\hline 59 & R Superior Frontal & 9 & 0.3 & 21 & 41 & 36 \\
\hline 60 & L Superior Frontal & 9 & 0.3 & -24 & 46 & 30 \\
\hline \multirow[t]{2}{*}{61} & R Middle Frontal & 8 & 0.2 & 26 & 24 & 39 \\
\hline & \multicolumn{6}{|l|}{ Temporal Lobe } \\
\hline \multirow[t]{2}{*}{62} & R Middle Temporal & 37 & 0.2 & 44 & -63 & 3 \\
\hline & \multicolumn{6}{|l|}{ Parietal Lobe } \\
\hline 63 & R Supramarginal & 40 & 0.6 & 52 & -43 & 34 \\
\hline
\end{tabular}

See Table 1 for footnote. 
primarily in the right hemisphere (e.g., right medial frontal gyrus, right superior frontal gyrus, bilateral inferior frontal gyrus, and right fusiform), and are consistent with previously reported findings (Tulving et al., 1994; Clark et al., 1998; Martin, 1999).

However, unlike the famous faces, the novel unfamiliar faces did not show increased activity in the hippocampus or temporofrontal region when contrasted with the NL faces. There is a striking absence of activation in these regions for comparisons involving NL versus FO retrieval and encoding versus retrieval of unfamiliar faces. Instead, increased frontal and parietal lobe activations were observed when NL and FO faces are compared. Frontal activations are commonly reported during episodic retrieval conditions and for novel stimuli (as discussed above). Encoding of unfamiliar faces showed increased activation in a medial and posterior parietal area likely related to the structural encoding task (i.e., rating of attractiveness). In contrast, recognition of newly learned faces produced greater activity than the encoding phase in the right supramarginal gyrus. Activation in this region could represent a temporary storage function of the parietal lobes in mediating recent episodic retrieval operations (Jonides et al., 1998).

Our neuroimaging findings could not be accounted for by behavioral variability because accuracy and reaction times were comparable in recognizing FF versus NL faces. Behavioral differences were observed with foil stimuli, which were rejected more slowly, but with a higher rate of accuracy than the FF and NL faces. By using an event-related fMRI design (D'Esposito et al., 1999), however, we were able to base our functional images solely on correct trials, thereby eliminating the problems in interpreting brain maps when behavioral differences are observed in blocked trial design experiments.

\section{Summary}

Our overall findings suggest a common neural network for longterm retrieval of famous faces. This network includes the temporofrontal region and the mesial temporal lobe, including the hippocampus and parahippocampus. This pattern of activation is consistent with other studies of long-term semantic and episodic memory (Fink et al., 1996; Maguire and Mummery, 1999). The relative degree of activation of the left and/or right side of this network may vary depending on different task and stimulus dimensions, which as yet remain poorly understood.

\section{REFERENCES}

Albert MS, Butters N, Levin J (1979) Temporal gradients in the retrograde amnesia of patients with alcoholic Korsakoff's disease. Arch Neurol 36:211-216.

Bandettini PA, Wong EC, Hinks RS, Tikofsky RS, Hyde JS (1992) Time course EPI of human brain function during task activation. Magn Reson Med 25:390-397.

Barr WB, Goldberg E, Wasserstein J, Novelly RA (1990) Retrograde amnesia following unilateral temporal lobectomy. Neuropsychologia 28:243-255.

Bruce V, Young A (1986) Understanding face recognition. Br J Psychol 77:305-327.

Burton AM, Bruce V, Johnston RA (1990) Understanding face recognition with an interactive activation model. Br J Psychol 81:361-380.

Clark VP, Maisog JM, Haxby JV (1998) fMRI study of face perception and memory using random stimulus sequences. J Neurophysiol 79:3257-3265.

Cohen NJ, Ryan J, Hunt C, Romine L, Wszalek T, Nash C (1999) Hippocampal system and declarative (relational) memory: summarizing the data from functional neuroimaging studies. Hippocampus 9:83-98.
Cox RW (1996) AFNI: software for analysis and visualization of functional magnetic resonance neuroimages. Comput Biomed Res 29:162-173.

D'Esposito M, Zarahn E, Aguirre GK (1999) Event-related functional MRI: implications for cognitive psychology. Psychol Bull 125:155-164. Damasio H, Grabowski TJ, Tranel D, Hichwa RD, Damasio AR (1996) A neural basis for lexical retrieval. Nature 380:499-505.

Evans JJ, Heggs AJ, Antoun N, Hodges JR (1995) Progressive prosopagnosia associated with selective right temporal lobe atrophy. A new syndrome? Brain 118:1-13.

Fink GR, Markowitsch HJ, Reinkemeier M, Bruckbauer T, Kessler J, Heiss WD (1996) Cerebral representation of one's own past: neural networks involved in autobiographical memory. $\mathrm{J}$ Neurosci 16:4275-4282.

Ghaem O, Mellet E, Crivello F, Tzourio N, Mazoyer B, Berthoz A, Denis M (1997) Mental navigation along memorized routes activates the hippocampus, precuneus, and insula. NeuroReport 8:739-744.

Grady CL, McIntosh AR, Horwitz B, Maisog JM, Ungerleider, LG, Mentis MJ, Pietrini P, Schapiro MB, Haxby JV (1995) Age-related reductions in human recognition memory due to impaired encoding. Science 269:218-221.

Greene JD, Hodges JR (1996) Identification of famous faces and famous names in early Alzheimer's disease. Relationship to anterograde episodic and general semantic memory. Brain 119:111-128.

Hanley JR, Pearson NA, Young AW (1990) Impaired memory for new visual forms. Brain 113:1131-1148.

Harris DM, Kay J (1995) I recognize your face but I can't remember your name: is it because names are unique? Br J Psychol 86:345-358.

Haxby JV, Ungerleider LG, Horwitz B, Maisog JM, Rapoport SI, Grady CL (1996) Face encoding and recognition in the human brain. Proc Natl Acad Sci USA 93:922-927.

Jonides J, Schumacher EH, Smith EE, Koeppe RA, Awh E, ReuterLorenz PA, Marshuetz C, Willis CR (1998) The role of parietal cortex in verbal working memory. J Neurosci 18:5026-5034.

Kapur N, Friston KJ, Young A, Frith CD, Frackowiak RS (1995) Activation of human hippocampal formation during memory for faces: a PET study. Cortex 31:99-108.

Kelley WM, Miezin FM, McDermott KB, Buckner RL, Raichle, ME, Cohen NJ, Ollinger JM, Akbudak E, Conturo TE, Snyder AZ, Petersen, SE (1998) Hemispheric specialization in human dorsal frontal cortex and medial temporal lobe for verbal and nonverbal memory encoding. Neuron 20:927-936.

Keren D, Peleg S, Brada R (1988) Image sequence enhancement using sub-pixel displacements. IEEE Conference on Computer Vision and Pattern Recognition, Ann Arbor, MI, June.

Kroll NE, Markowitsch HJ, Knight RT, Von Cramon DY (1997) Retrieval of old memories: the temporofrontal hypothesis. Brain 120:1377-1399.

Maguire EA, Mummery CJ (1999) Differential modulation of a common memory retrieval network revealed by positron emission tomography. Hippocampus 9:54-61.

Maguire EA, Frackowiak RSJ, Frith CD (1997) Recalling routes around London: activation of the right hippocampus in taxi drivers. J Neurosci 17:7103-7110.

Markowitsch HJ (1995) Which brain regions are critically involved in the retrieval of old episodic memory? Brain Res Rev 21:117-127.

Marslen-Wilson WD, Teuber HL (1975) Memory for remote events in anterograde amnesia: recognition of public figures from news photographs. Neuropsychologia 13:353-364.

Martin A (1999) Automatic activation of the medial temporal lobe during encoding: lateralized influences of meaning and novelty. Hippocampus 9:62-70.

Mummery CJ, Patterson K, Wise RJS, Vandenberghe R, Price CJ, Hodges JR (1999) Disrupted temporal lobe connections in semantic dementia. Brain 122:61-73.

Nadel L, Moscovitch M (1997) Memory consolidation, retrograde amnesia and the hippocampal complex. Curr Opin Neurobiol 7:217-227.

Nyberg L, McIntosh AR, Cabeza R, Habib R, Houle S, Tulving E (1996) General and specific brain regions involved in encoding and retrieval of events: what, where, and when. Proc Natl Acad Sci USA 93:11280-11285.

Oldfield RC (1971) The assessment and analysis of handedness: The Edinburgh Inventory. Neuropsychologia 9:97-113.

Reinkemeier M, Markowitsch HJ, Rauch M, Kessler J (1997) Differen- 
tial impairments in recalling people's names: a case study in search of neuroanatomical correlates. Neuropsychologia 35:677-684.

Rempel-Clower NL, Zola SM, Squire LR, Amaral DG (1996) Three cases of enduring memory impairment after bilateral damage limited to the hippocampal formation. J Neurosci 16:5233-5255.

Rolls ET (1992) Neurophysiological mechanisms underlying face processing within and beyond the temporal cortical visual areas. Philos Trans R Soc Lond B Biol Sci 335:11-20.

Sergent J, Ohta S, MacDonald B (1992) Functional neuroanatomy of face and object processing. Brain 115:15-36.

Squire LR, Alvarez P (1995) Retrograde amnesia and memory consolidation: a neurobiological perspective. Curr Opin Neurobiol 5:169-177.

Talairach J, Tournoux P (1988) Co-planar stereotaxic atlas of the human brain. New York: Thieme.

Tempini ML, Price CJ, Josephs O, Vandenberghe R, Cappa SF, Kapur N, Frackowiak RS (1998) The neural systems sustaining face and propername processing. Brain 121:2103-2118.

Thompson-Schill SL, D'Esposito M, Aguirre GK, Farah MJ (1997) Role of left inferior prefrontal cortex in retrieval of semantic knowledge: a reevaluation. Proc Natl Acad Sci USA 94:14792-14797.

Tranel D, Damasio H, Damasio AR (1997) A neural basis for the retrieval of conceptual knowledge. Neuropsychologia 35:1319-1327.

Tulving E, Markowitsch HJ, Kapur S, Habib R, Houle S (1994) Novelty encoding networks in the human brain: positron emission tomography data. NeuroReport 5:2525-2528.

Vandenberghe R, Dupont P, De Bruyn B, Bormans G, Michiels J, Mortelmans L, Orban GA (1996) The influence of stimulus location on the brain activation pattern in detection and orientation discrimination: a PET study of visual attention. Brain 119:1263-1276.

Warrington EK, James M (1967) An investigation of facial recognition in patients with unilateral cerebral lesions. Cortex 3:317-326.

Warrington EK, McCarthy RA (1988) The fractionation of retrograde amnesia. Brain Cogn 7:184-200.

Young AW, Newcombe F, de Haan EH, Small M, Hay DC (1993) Face perception after brain injury. Selective impairments affecting identity and expression. Brain 116:941-959. 